\title{
High-Quality Genome Sequence Resource of a Rice False Smut Fungus Ustilaginoidea virens Isolate, UV-FJ-1
}

\author{
Jiandong Bao, ${ }^{1}$ Rong Wang, ${ }^{2}$ Shilei Gao, ${ }^{1}$ Zhe Wang, ${ }^{1}$ Yu Fang, ${ }^{2}$ Lv Wu, ${ }^{3}$ and Mo Wang ${ }^{1,2, \dagger}$ \\ ${ }^{1}$ Fujian University Key Laboratory for Plant-Microbe Interaction, College of Life Sciences, Fujian \\ Agriculture and Forestry University, Fuzhou, 350002, China \\ ${ }^{2}$ State Key Laboratory of Ecological Pest Control for Fujian and Taiwan Crops, Plant Immunity Center, \\ Fujian Agriculture and Forestry University, Fuzhou, Fujian 350002, China \\ ${ }^{3}$ Gongzhuling Sub-center for New Plant Variety Tests, Ministry of Agriculture and Rural Affairs, Jilin \\ Academy of Agricultural Sciences, Changchun, 136100, China
}

\begin{abstract}
Ustilaginoidea virens is the fungal pathogen causing rice false smut, resulting in not only yield lost but also grain pollution with toxic mycotoxins. Here we deployed PacBio Sequel II HIFIread sequencing technology to generate a near-complete genome assembly for the $U$. virens isolate UV-FJ-1 (38.48 Mb), which was isolated from Fujian province, China. The genome assembly contains 116 contigs with $\mathrm{N}_{50}$ of $0.65 \mathrm{Mb}$ and a maximum length of $2.10 \mathrm{Mb}$, and the genome completeness is $\geq 98 \%$ assessed by benchmarking universal single-copy orthologs (BUSCOs) and the mapping rate of Illumina short reads. Excluding $35.78 \%$ repeat sequences, we identified a total of 7,164 protein-coding genes, of which 5,818 were functionally annotated and 223 encode putative effector proteins. Moreover, 21 secondary metabolite biosynthesis gene clusters were found in UV-FJ-1 genome. Taken together, this high-quality genome assembly and gene annotation resource will provide a better insight for characterizing the biological and pathogenic mechanisms of $U$. virens.
\end{abstract}

\section{Genome Announcement}

Rice false smut, which was first reported in southern India in the 1878 (Cooke 1878), has recently emerged as the most devastating rice grain disease, because it not only results in considerable yield loss with annually increasing incidence area, but also contaminates rice grains by poisonous mycotoxins threatening the health of both humans and livestock (Sun et al. 2020). Rice false smut is caused by an ascomycete fungal pathogen, Ustilaginoidea virens (U. virens, teleomorph: Villosiclava virens), which is evolutionarily close to the entomopathogenic Metarhizium, also known as green muscardine fungus (Zhang et al. 2014). U. virens is heterothallic and performs sexual reproduction under field and laboratory conditions (Sun et al. 2020; Yu et al. 2015). In the field, both the chlamydospores and ascospores of $U$. virens, generated from asexual and sexual cycles, respectively, could produce secondary conidia and serve as inocula (Fan et al. 2016). As a flowerinfecting pathogen, $U$. virens starts to infect rice spikelets at the late rice booting stage, and ultimately embraces all the organs and produces a smut ball during grain filling stage (Ashizawa et al. 2012; Hu et al. 2014).

Currently, the genome sequences of three U. virens isolates, UV-8b, IPU010, and UV-Gvt, originated from China, Japan, and India, respectively, are publicly available on the Assembly Database of NCBI (Kumagai et al. 2016; Pramesh et al. 2020; Zhang et al. 2014). The genome sizes of these $U$. virens isolates range from 26.97 to $38.06 \mathrm{Mb}$.

\footnotetext{
${ }^{\dagger}$ Corresponding author: M. Wang; wangmo108@163.com
}

The author(s) declare no conflict of interest.

Accepted for publication 17 March 2021.

\section{Funding}

This work was supported by the grants from FAFU Foundation for Distinguished Young Scholars (xjq201708) and Youth Program of National Natural Science Foundation of China (31701777) to M. Wang.

\section{Keywords}

genome, PacBio Sequel II HIFl-reads, rice false smut, Ustilaginoidea virens 
Table 1. Statistics of genome assembly of the Ustilaginoidea virens isolates

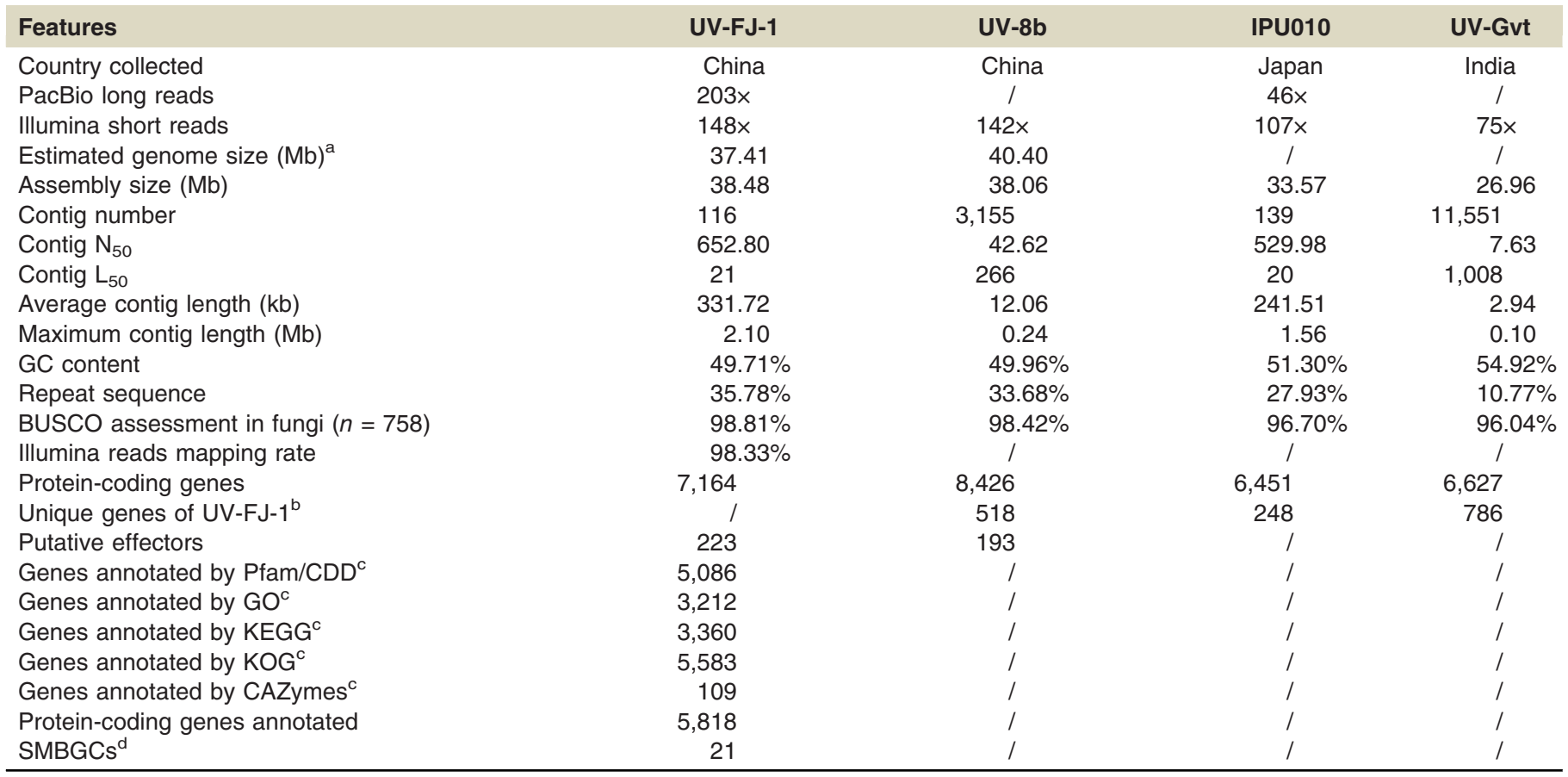

${ }^{a}$ Genome size was estimated by KmerGenie (best $\mathrm{k}=107$ ) using Illumina short reads.

b The numbers indicate the UV-FJ-1 genes hitting less than $80 \%$ coverage by Blastn or absent in the genome of UV-8b, IPU010, or UV-Gvt isolate.

${ }^{c}$ Pfam and conserved domain database (CDD) annotations were conducted by InterProScan v5.47-82.0; gene ontology (GO), Kyoto encyclopedia of genes and genomes (KEGG), eukaryotic orthologous groups (KOG), and carbohydrate-active enzymes (CAZymes) annotations were conducted by eggNOG-mapper v2 (detailed in Fig. 1C).

d Twenty-one secondary metabolite biosynthesis gene clusters (SMBGCs) were analyzed by fungal version of antiSMASH v5.2.0, which includes six nonribosomal peptide synthetases (NRPSs), seven NPRS-like, four terpenes, and four type I polyketide synthases.

Here, we isolated a U. virens isolate in Fujian province of China, named UV-FJ-1. Its genomic DNA for sequencing was prepared as below: UV-FJ-1 mycelium grown on potato dextrose agar medium for 10 days was transferred to $300 \mathrm{ml}$ of liquid medium containing peeled potatoes at $200 \mathrm{~g} / \mathrm{liter}$ and glucose at $20 \mathrm{~g} /$ liter; after cultured for 12 days with shaking $(120 \mathrm{rpm})$ at $28^{\circ} \mathrm{C}$, the mycelium was collected for DNA extraction with the CTAB method. Its genome was sequenced by PacBio Sequel II HIFI-read sequencing technology at Annoroad Gene Technology Co. Ltd. (Beijing, China) combining Illumina short-read sequencing technology at the Novogene Bioinformatics Technology Co. Ltd (Beijing, China). The PacBio Sequel II sequencing platform generated $7.82 \mathrm{~Gb}\left(\sim 203 \times ; \mathrm{N}_{50}=11.49 \mathrm{~kb}\right)$ subreads, meanwhile the Illumina HiSeq 2000 sequencing platform produced $5.71 \mathrm{~Gb}(\sim 148 \mathrm{x})$ 150-bp paired-end adaptors with 350-bp insertion size library (Table 1). Genome size of UV-FJ-1 was estimated by KmerGenie (http://kmergenie.bx.psu.edu/) v1.7051 based on the k-mer distribution of the lllumina short reads. The predicted assembly size was $37,405,008$ bp at best $k=107$ (Table 1).

NextDenovo v2.3.1 (https://github.com/Nextomics/NextDenovo), a string graph-based de novo assembler developed by Nextomics, was employed to generate a draft genome assembly using pure PacBio long reads (seed reads were cut off at 14,239 bp). Subsequently, the draft genome assembly was polished by NextPolish v1.3.1 (https://github.com/Nextomics/NextPolish) to correct base errors using both of PacBio long reads and Illumina short reads. Finally, we obtained a $38.48 \mathrm{Mb}$ polished genome assembly consisting of 116 contigs with a contig $N_{50}$ of $0.65 \mathrm{Mb}\left(L_{50}=21\right)$, average contig length of $0.33 \mathrm{Mb}$, and maximum contig length of $2.10 \mathrm{Mb}$ (Table 1, Fig. 1A). The assembly size of UV-FJ-1 genome was very similar to the estimated genome size (38.48 versus $37.41 \mathrm{Mb})$. Compared with the previously reported genome assemblies (UV-8b, IPU010, and UV-Gvt), our assembly was with the biggest size and fewest contigs, thus with the largest value of contig $\mathrm{N}_{50}$ as well as the average and maximum contig length (Table 1).

The genome completeness of UV-FJ-1 was assessed by BUSCO v4.14 (Seppey et al. 2019) using benchmarking universal single-copy orthologs (BUSCOs) at Ascomycota ( $n=1,706)$, fungi ( $n=758)$, and Sordariomycetes ( $n=3,817$ ) levels. We identified 1,670 (97.89\%), 749 (98.81\%), and $3,762(98.55 \%)$ complete BUSCOs for Ascomycota, fungi, and Sordariomycetes datasets, respectively (Fig. 1B). Compared with the other three published $U$. virens genomes, the BUSCO 

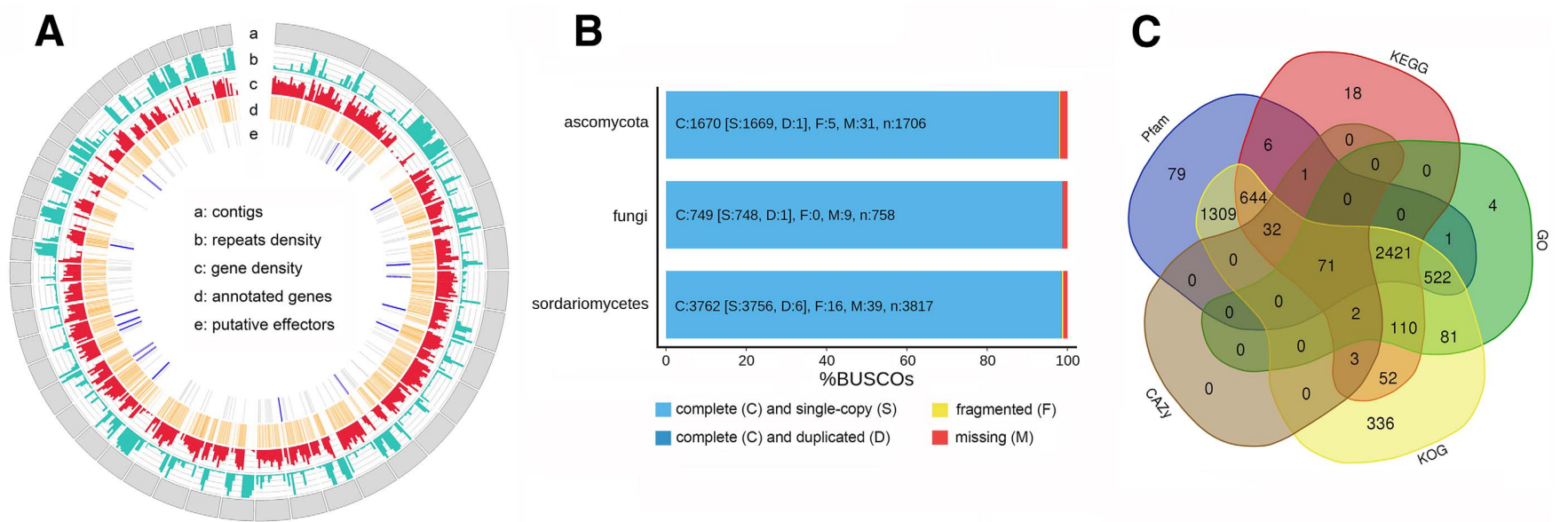

Fig. 1. Schematic diagrams of the sequencing, assembly and annotation of Ustilaginoidea virens isolate UV-FJ-1 genome. A, Genome features of UV-FJ-1 shown by Circos diagram (http://circos.ca/). a: the contigs >100 kb; b: repeats calculated by sliding window analysis (50kb); c: the density of predicted genes; d: genes with functional annotation; and e: putative effector proteins, among which the UV-FJ-1-specific effectors compared with Uv-8b are highlighted with blue. B, The completeness of UV-FJ-1 genome assembly was assessed by BUSCO v4.1.4 with single-copy orthologs from OrthoDB v10 at ascomycota $(n=1,706)$, fungi $(n=758)$, and sordariomycetes $(n=3,817)$ levels. C, Gene annotation shown by venn diagram (http://bioinformatics.psb.ugent.be/webtools/Venn/). Pfam and CDD annotations were conducted by InterProScan v5.47-82.0, and GO, KEGG, KOG, and CAZy annotations were performed by eggNOG-mapper v2.

assessment results at fungi level indicate that the assembly of UV-FJ-1 genome has the highest completeness (98.81\%), followed by UV-8b (98.42\%), IPU010 (96.70\%), and UV-Gvt (96.04\%) (Table 1). The genome completeness was also evaluated by mapping rate of the lllumina short reads, which were performed using BWA v0.7.17 (https://github.com/lh3/bwa), and a total of 98. $33 \%$ reads were properly mapped to the genome assembly (Table 1 ). Taken together, these results indicate that the genome completeness of $U$. virens isolate UV-FJ-1 was over $98 \%$.

Moreover, RepeatModeler v2.01 (http://www.repeatmasker.org/RepeatModeler/) was used to generate a de novo repeats library, which was applied to perform repeat masking with RepeatMasker v4.1.1 (http://www.repeatmasker.org/), and 35.78\% repetitive sequences in the genome assembly of UV-FJ-1 was found (Fig. 1A, Table 1). Then the repeat-masked genome sequence was subjected to gene prediction by Fgenesh (Solovyev et al. 2006) (organism = Ustilago), and 7,164 protein-coding genes were identified in UV-FJ-1, which is less than 8,426 in UV-8b but more than 6,451 and 6,627 in IPU010 and UV-Gvt, respectively (Fig. 1A, Table 1). The isolatespecific genes of UV-FJ-1 are 518, 248, and 786 when compared with UV-8b, IPU010, and UV-Gvt, respectively, indicating $\sim 90 \%$ genes are conserved among different $U$. virens isolates (Table 1). UV-FJ-1 possesses 223 putative effectors (characterized by the protein length $\leq 400$ amino acids, containing signal peptide and $\geq 4$ cysteine residues, and without any transmembrane domain) (Zhang et al. 2014), of which 31 are isolate-specific compared with UV-8b (Fig. 1A). Furthermore, a total of 5,818 genes $(81.21 \%)$ in UV-FJ-1 were annotated by either InterProScan v5.47.82 (https://github.com/ebi-pf-team/interproscan) or eggNOG-mapper v2 (http://eggnog-mapper.embl. $\mathrm{de} /$ ) with databases including Pfam/conserved domain database (CDD) $(5,086)$, gene ontology (GO; 3,212), Kyoto encyclopedia of genes and genomes (KEGG; 3,360), eukaryotic orthologous groups (KOG; 5,583), and carbohydrate-active enzymes (CAZymes; 109) (Table 1, Fig. 1A and C). Secondary metabolite biosynthesis gene clusters (SMBGCs) were analyzed by fungal version of antiSMASH v5.2.0 (https://fungismash.secondarymetabolites.org/), and 21 SMBGCs including six nonribosomal peptide synthetases (NRPSs), seven NPRS-like, four terpenes and four type I polyketide synthases (T1PKSs) were identified (Table 1).

The whole genome sequence data including the gene prediction and annotation information reported in this paper have been deposited in the Genome Warehouse (http://bigd.big.ac.cn/gwh/) in National Genomics Data Center, Beijing Institute of Genomics (China National Center for Bioinformation), Chinese Academy of Sciences, under accession number GWHASIW000000000.1 (BioProject PRJCA003902). 


\section{Literature Cited}

Ashizawa, T., Takahashi, M., Arai, M., and Arie, T. 2012. Rice false smut pathogen, Ustilaginoidea virens, invades through small gap at the apex of a rice spikelet before heading. J. Gen. Plant Pathol. 78:255-259.

Cooke, M. C. 1878. Some extra-European fungi. Grevillea 7:13-15.

Fan, J., Yang, J., Wang, Y.-Q., Li, G.-B., Li, Y., Huang, F., and Wang, W.-M. 2016. Current understanding on Villosiclava virens, a unique flower-infecting fungus causing rice false smut disease. Mol. Plant Pathol. 17:1321-1330.

Hu, M., Luo, L., Wang, S., Liu, Y., and Li, J. 2014. Infection processes of Ustilaginoidea virens during artificial inoculation of rice panicles. Eur. J. Plant Pathol. 139:67-77.

Kumagai, T., Ishii, T., Terai, G., Umemura, M., Machida, M., and Asaib, K. 2016. Genome sequence of Ustilaginoidea virens IPU010, a rice pathogenic fungus causing false smut. Genome Announc. 4:e00306-e00316.

Pramesh, D., Prasannakumar, M. K., Muniraju, K. M., Mahesh, H. B., Pushpa, H. D., Manjunatha, C., Saddamhusen, A., Chidanandappa, E., Yadav, M. K., Kumara, M. K., Sharanabasav, H., Rohith, B. S., Banerjee, G., and Das, A. J. 2020.
Comparative genomics of rice false smut fungi Ustilaginoidea virens Uv-Gvt strain from India reveals genetic diversity and phylogenetic divergence. 3 Biotech 10:342.

Seppey, M., Manni, M., and Zdobnov, E. M. 2019. BUSCO: Assessing genome assembly and annotation completeness. Methods Mol. Biol. 1962:227-245.

Solovyev, V., Kosarev, P., Seledsov, I., and Vorobyev, D. 2006. Automatic annotation of eukaryotic genes, pseudogenes and promoters. Genome Biol. 7:S10.11-12.

Sun, W., Fan, J., Fang, A., Li, Y., Tariqjaveed, M., Li, D., Hu, D., and Wang, W.-M. 2020. Ustilaginoidea virens: Insights into an emerging rice pathogen. Annu. Rev. Phytopathol. 58:363-385.

Yu, J., Sun, W., Yu, M., Yin, X., Meng, X., Zhao, J., Huang, L., Huang, L., and Liu, Y. 2015. Characterization of mating-type loci in rice false smut fungus Villosiclava virens. FEMS Microbiol. Lett. 362:fnv014.

Zhang, Y., Zhang, K., Fang, A., Han, Y., Yang, J., Xue, M., Bao, J., Hu, D., Zhou, B., Sun, X., Li, S., Wen, M., Yao, N., Ma, L.-J., Liu, Y., Zhang, M., Huang, F., Luo, C., Zhou, L., Li, J., Chen, Z., Miao, J., Wang, S., Lai, J., Xu, J.-R., Hsiang, T., Peng, Y.-L., and Sun, W. 2014. Specific adaptation of Ustilaginoidea virens in occupying host florets revealed by comparative and functional genomics. Nat. Commun. 5:3849. 